\title{
Learning Rate Updating Methods Applied to Adaptive Fuzzy Equalizers for Broadband Power Line Communications
}

\author{
Moisés V. Ribeiro \\ Department of Communications, State University of Campinas, 13083970 São Paulo, Brazil \\ Email: mribeiro@ieee.org
}

Received 1 September 2003; Revised 31 May 2004

\begin{abstract}
This paper introduces adaptive fuzzy equalizers with variable step size for broadband power line (PL) communications. Based on delta-bar-delta and local Lipschitz estimation updating rules, feedforward, and decision feedback approaches, we propose singleton and nonsingleton fuzzy equalizers with variable step size to cope with the intersymbol interference (ISI) effects of PL channels and the hardness of the impulse noises generated by appliances and nonlinear loads connected to low-voltage power grids. The computed results show that the convergence rates of the proposed equalizers are higher than the ones attained by the traditional adaptive fuzzy equalizers introduced by J. M. Mendel and his students. Additionally, some interesting BER curves reveal that the proposed techniques are efficient for mitigating the above-mentioned impairments.
\end{abstract}

Keywords and phrases: power line communications, broadband applications, nonlinear equalization, fuzzy systems, learning rate updating, impulse noises.

\section{INTRODUCTION}

In recent years, the increased demand for fast Internet accesses and new multimedia services, the development of new and feasible signal processing techniques associated with faster and low-cost digital signal processors, and the deregulation of the telecommunications market have placed considerable emphasis on the value of investigating hostile media, such as power line (PL) channels $[1,2,3]$, for high-rate transmissions. A considerable body of research has given much attention to indoor (last-meter, residential, or intrabuilding) and outdoor (last-miles or local area networks and rural networks) PL environments for broadband applications $[2,3,4,5,6,7,8]$.

For last-miles environments, it has been demonstrated that PL channels are as good as telephone and cable TV channels for the transmission of broadband contents $[1,2$, $3,9,10]$. The capacity of PL channels for last-miles applications can surpass $450 \mathrm{Mbps}$ [9]. Modems with bit rates higher than $10 \mathrm{Mbps}$ are nowadays offered by some companies. Nevertheless, a new generation of power line communications (PLC) modems that exceed $50 \mathrm{Mbps}$ is appearing [10].

Such improvement demands, however, some special schemes or solutions for coping with the following problems in the physical layer: (a) the considerable differences between PL networks; and (b) the hostile properties of
PL channels, such as attenuation proportional to high frequencies and long distances, high-power impulse noise occurrences, and strong intersymbol interference (ISI) effects.

Equalization techniques are so far widely employed to cope with ISI effects $[11,12,13]$. Among linear and nonlinear equalization techniques available in the literature, adaptive fuzzy equalizers are pointed out as good candidates to tackle nonlinear features of the impulse noises and the severity of the ISI effects, as postulated in $[12,13]$.

Aiming at the development of nonlinear equalization techniques based on adaptive fuzzy systems for broadband PLCs, this paper introduces singleton (S) and nonsingleton (NS) fuzzy [14] equalizers with variable step size. Delta-bardelta (DBD) learning rule [15] and local Lipschitz estimation (LLE) [16] are the methods chosen to tune the individual step size of each free parameter of adaptive fuzzy equalizers. The proposed fuzzy techniques emerge as interesting solutions for the equalization of PL channels and mitigation of impulse noises. In fact, PL channels change periodically, and periodic PL channel equalizations with shorter training sequences are required to achieve high bit rates. The findings reveal that such new techniques show higher convergence rates than traditional adaptive fuzzy equalizers introduced by J. M. Mendel and his students. Additionally, the proposed techniques are able to equalize outdoor PL channels and also mitigate impulse noises. 
The rest of the paper is organized as follows. Section 2 gives a brief overview of PLCs in low-voltage grids. Section 3 focuses on the proposed techniques. Section 4 shows some results of numerical simulations. Finally, Section 5 states some concluding remarks.

\section{POWER LINE COMMUNICATIONS IN LOW-VOLTAGE GRIDS: AN OVERVIEW}

Although not built for communication applications, the electrical distribution circuits have been used for these purposes since 1838. In the 1980s, several signal processing techniques, such as error control coding and modulation techniques, started to be implemented in hardware to achieve transmission rates up to $14.4 \mathrm{kbps}$. At the same time the CELENEC standard emerged in Europe to address typical narrowband applications at rates up to $144 \mathrm{kbps}$ over distances around $500 \mathrm{~m}$ and maximum signal power of $5 \mathrm{~mW}$ [1]. Nowadays, PL channels are used in frequency range between 1 and $30 \mathrm{MHz}$ for broadband indoor and outdoor applications. In this context, regulatory framework to harmonize the coexistence between PLC systems and radio services is mandatory since the radio services has been previously allocated in the frequency range between 1 and $30 \mathrm{MHz}$. Recent investigations supporting PLC and radio services interoperability, coexistence, and electromagnetic compatibility estimate that the power spectral density (PSD) of the data signal transmitted on PLs must range from $-79 \mathrm{dBV}^{2} / \mathrm{Hz}$ to $-50 \mathrm{dBV}^{2} / \mathrm{Hz}$ [11]. The frequency response of the low-voltage distribution network (LVDN) is given by [6]

$$
\begin{aligned}
H(f)=\sum_{i=1}^{M}\left|g_{i}(f)\right| \exp \left(\varphi_{g_{i}}(f)\right) \\
\quad \times \exp \left[-\left(a_{0}+a_{1} f^{k}\right)\right] \exp \left(-2 \pi f \tau_{i}\right),
\end{aligned}
$$

where $g_{i}(f)$ denotes the weighting factor in the ith multipath; $\exp \left[-\left(a_{0}+a_{1} f^{k}\right)\right]$ is the attenuation term; $\exp \left(-2 \pi f \tau_{i}\right)$ is the delay portion in the $i$ th multipath; and $M$ is the number of multipaths. Figure 1 illustrates the frequency response of three PL channels.

For the frequency band from 1 to $30 \mathrm{MHz}$, the noise is modeled as an additive contribution and expressed by [7]

$$
\begin{aligned}
\eta(n)= & \eta_{\mathrm{bkgr}}(n)+\eta_{\mathrm{pb}}(n)+\eta_{\mathrm{pa}}(n) \\
& +\eta_{\mathrm{ps}}(n)+\eta_{\mathrm{imp}}(n),
\end{aligned}
$$

where $\eta_{\mathrm{bkgr}}(n)$ is the background noise; $\eta_{\mathrm{nb}}(n)$ is a narrowband noise; $\eta_{\mathrm{pa}}(n)$ is a periodical impulse noise asynchronous to the fundamental component of power system; $\eta_{\mathrm{ps}}(n)$ is a periodic impulse noise synchronous to the fundamental component of power system; and finally $\eta_{\text {imp }}(n)$ is an asynchronous impulse noise. Figure 2 shows a typical noise in the PL channel generated as in [7] in the frequency band between 2 and $3 \mathrm{MHz}$. The PSDs of the colored background and impulse noises are equal to $-130 \mathrm{dBV}^{2} / \mathrm{Hz}$ and

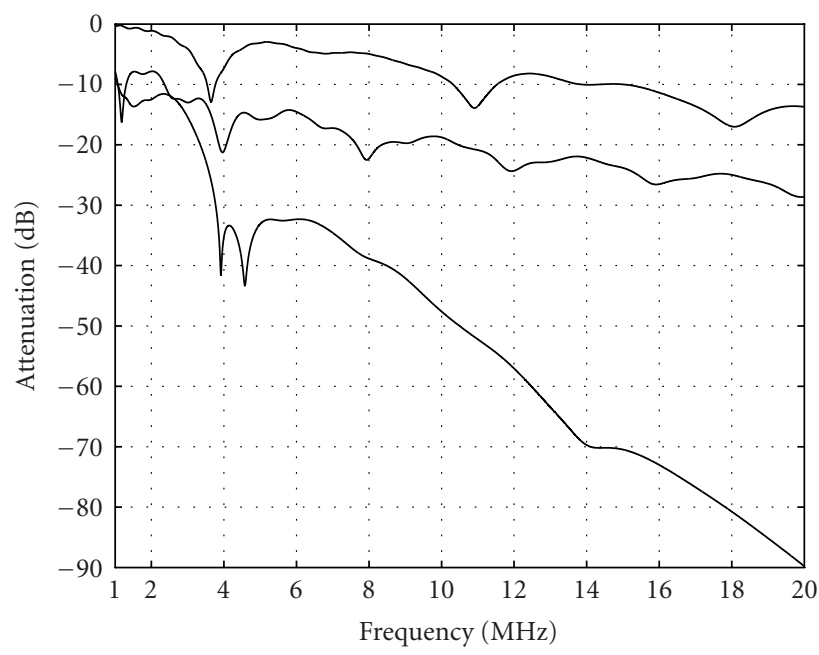

FIGURE 1: Frequency response of three PL channels.

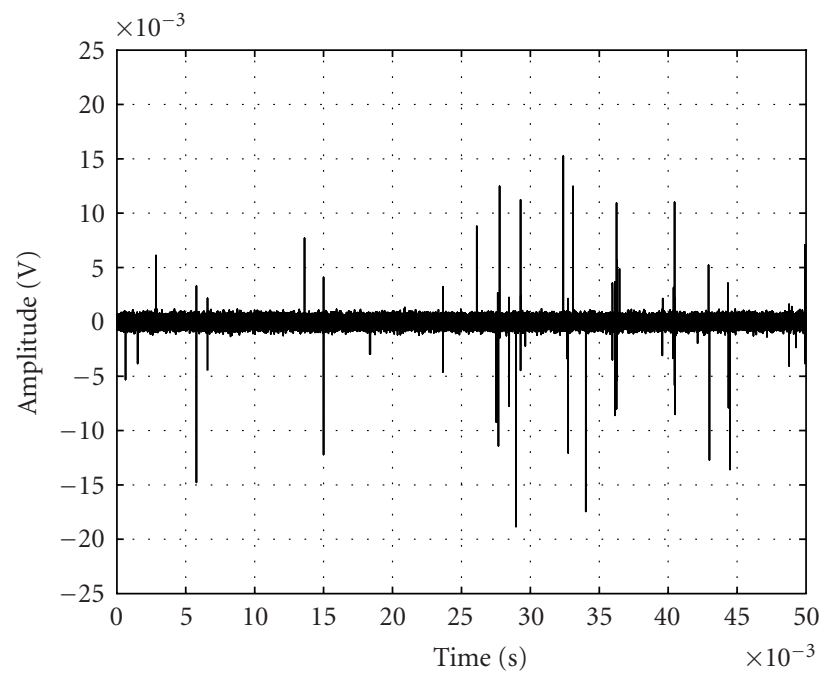

FIgUre 2: Additive noise in LVDN.

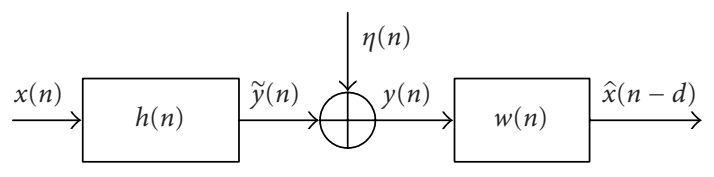

FIgURE 3: Discrete time model of PL digital communication system.

$-110 \mathrm{dBV}^{2} / \mathrm{Hz}$, respectively. The maximum amplitude of the impulse noises, shown in Figure 2, is lower than $20 \mathrm{mV}$. However, this value can be higher than $100 \mathrm{mV}$.

A discrete time model of a digital communication system for PLC that takes into account the effect of ISI and the presence of additive noise is portrayed in Figure 3. 
The symbol-spaced channel output is

$$
\begin{aligned}
y(n) & =\tilde{y}(n)+\eta(n) \\
& =\sum_{k=0}^{L_{h}-1} h(k) x(n-k)+\eta(n), \quad-\infty \leq k \leq \infty,
\end{aligned}
$$

where the transmitted sequence $x(n)$ is taken from $\{-1,+1\}$ and it is assumed to be an equiprobable and independent sequence with $E\{x(n-k) x(n-l)\}=\sigma_{x}^{2} \delta(k-l)$ and $E\{x(n)\}=$ 0 . $\{h(n)\}_{n=0}^{L_{h}-1}$ is the bandlimited, dispersive, and linear FIR $\mathrm{PL}$ channel model whose frequency response is expressed by (1). The additive impulse noise $\eta(n)$ is given by (2) and $\tilde{y}(n)$ denotes the noise-free channel output. The channel outputs observed by the linear equalizer $\{w(n)\}_{n=0}^{L_{w}-1}$ can be written as vector $\mathbf{y}(n)=\left[\begin{array}{lll}y(n) & \cdots & y\left(n-L_{w}+1\right)\end{array}\right]^{T} \in$ $\mathfrak{R}^{L_{w}}$. The vector of the transmitted symbols that influence the equalizer decision is expressed by $\mathbf{x}(n)=$ $\left[x(n) \cdots x\left(n-L_{w}-L_{h}+1\right)\right]^{T}$. As a result, there are $n_{s}=$ $2^{L_{w}+L_{h}-1}$ possible combinations of the channel input sequence; and $n_{s}$ different values of the noise-free channel output vector $\tilde{\mathbf{y}}(n)=\left[\begin{array}{llll}\tilde{y}(n) & \cdots & \tilde{y}\left(n-L_{w}+1\right)\end{array}\right]^{T}$ are possible. Each of these noise-free channel output vector values is called a channel output state vector $\tilde{\mathbf{y}}_{j}, j=1, \ldots, n_{s}$, given by

$$
\tilde{\mathbf{y}}_{j}=\mathbf{H} \mathbf{x}_{j}
$$

where $\mathbf{x}_{j}=\left[x_{j}(n) \cdots x_{j}\left(n-L_{h}-L_{w}+1\right)\right]^{T}$ denotes the $j$ th input vector and $\mathbf{H}$ is a matrix channel impulse response given in the form of

$$
\mathbf{H}=\left[\begin{array}{cccccc}
h_{0} & h_{1} & \cdots & h_{L_{h}-1} & \cdots & 0 \\
0 & h_{0} & \cdots & h_{L_{h}-2} & \cdots & 0 \\
\vdots & \vdots & & & & \vdots \\
0 & 0 & h_{0} & \cdots & h_{L_{h}-2} & h_{L_{h}-1}
\end{array}\right]
$$

The equalizer output $\hat{x}(n-d)$ is a delayed form of the transmitted sequence.

Based on the single-input single-output (SISO) concept, the PL channels can be equalized by using two categories of adaptive equalization techniques, namely, sequence estimation and symbol decision. The optimal solution for sequence estimation is achieved by using maximum-likelihood sequence estimation (MLSE) [17]. The MLSE is implemented by using the Viterbi algorithm [18], which determines the estimated transmitted sequence $\{\hat{x}(n)\}_{n=0}^{\infty}$ when the cost function defined by

$$
J=\sum_{n=0}^{\infty} y(n)\left(\sum_{k=0}^{L_{h}-1} h(k) \hat{x}(n-k)\right)
$$

is minimized. Although this algorithm demands the highest computational cost, it provides the lowest error rate when the channel is known.
The optimal solution for symbol decision equalization is obtained from the Bayes probability theory [19]. The normalized optimal Bayesian equalizer (NOBE) is defined by

$$
\begin{aligned}
f_{b}(\mathbf{y}(n))= & \frac{1}{\sum_{\mathbf{y}_{k} \in \mathbf{C}_{d}} \exp \left(-\left\|\mathbf{y}(n)-\tilde{\mathbf{y}}_{k}\right\|^{2} / 2 \sigma_{n}^{2}\right)} \\
& \times\left\{\sum_{\mathbf{y}_{i} \in \mathbf{C}_{d}^{+}} \exp \left(\frac{-\left\|\mathbf{y}(n)-\tilde{\mathbf{y}}_{i}\right\|^{2}}{2 \sigma_{n}^{2}}\right)\right. \\
& \left.-\sum_{\mathbf{y}_{j} \in \mathbf{C}_{d}^{-}} \exp \left(\frac{-\left\|\mathbf{y}(n)-\tilde{\mathbf{y}}_{j}\right\|^{2}}{2 \sigma_{n}^{2}}\right)\right\},
\end{aligned}
$$

where the noise source is assumed to be zero mean additive white Gaussian with variance equal to $\sigma_{n}^{2}$; and $\mathbf{C}_{d}^{+}=\{\mathbf{y}(n) \mid$ $x(n-d)=+1\}$ and $\mathbf{C}_{d}^{-}=\{\mathbf{y}(n) \mid x(n-d)=-1\}$ make up the channel states matrix $\mathbf{C}_{d}=\mathbf{C}_{d}^{+} \cup \mathbf{C}_{d}^{-}=\left\{\tilde{\mathbf{y}}_{j}\right\}, 1 \leq j \leq n_{s}$.

Despite the optimality of the Bayesian equalizer, the clustering or channel estimation techniques used to estimate the channel output vector states demand prohibitive computational cost. The same problem is observed when an adaptive implementation of the Bayesian equalizer based on a backpropagation method [20] is performed to adjust the Bayesian free parameters.

\section{THE PROPOSED FUZZY EQUALIZERS}

Nonlinear equalization techniques based on computational intelligence have been widely applied to mitigate ISI effects in linear and nonlinear channels as well as to minimize the influence of non-Gaussian noises [12, 13, 14, 21, 22, 23, 24, 25, 26]. Among them, singleton type-1 fuzzy systems [12, 13, 14] are pointed out to be a good solution for ISI and impulse noise mitigations. In $[24,25]$, it was demonstrated that the NOBE is a particular case of a singleton type-1 fuzzy system and that its implementation as a fuzzy filter demands low computational complexity. A substantial lower computational complexity is achieved if the method suggested in [27] is applied.

As far as channel equalization is concerned, more complexity reduction is attained when a decision feedback (DF) structure $[28,29]$ is adopted to implement fuzzy equalizers. In this case, let the order of the feedback branch $L_{b}$ be equal to $L_{h}+L_{w}-d-1$, then the feedback vector can assume $n_{b}=2^{L_{b}}$ states. Thus, the channel states matrix $\mathbf{C}_{d}$ can be divided into $n_{b}$ subsets. The new positive and negative channel state matrices are given by

$$
\begin{aligned}
& \mathbf{C}_{d}^{++}=\{\mathbf{y}(n) \mid x(n-d)=+1 \cap \hat{x}(n-d)=+1\}, \\
& \mathbf{C}_{d}^{--}=\{\mathbf{y}(n) \mid x(n-d)=-1 \cap \hat{x}(n-d)=-1\} .
\end{aligned}
$$

As a result, the related number of states in $\mathbf{C}_{d}^{++}$and $\mathbf{C}_{d}^{--}$becomes equal to

$$
n_{n s}=\frac{n_{s}}{n_{b}}=2^{d}
$$




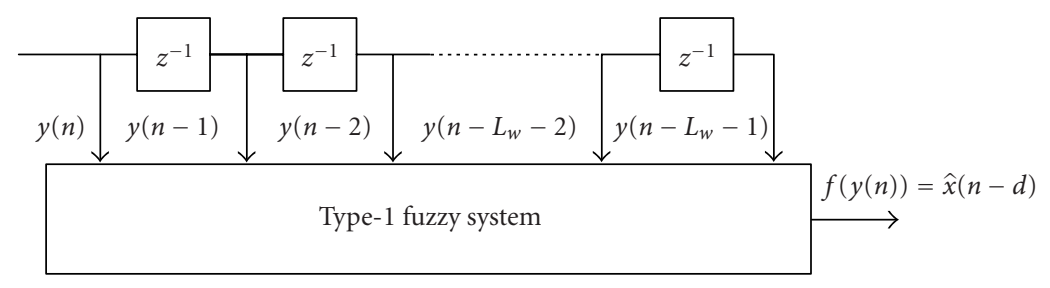

(a)

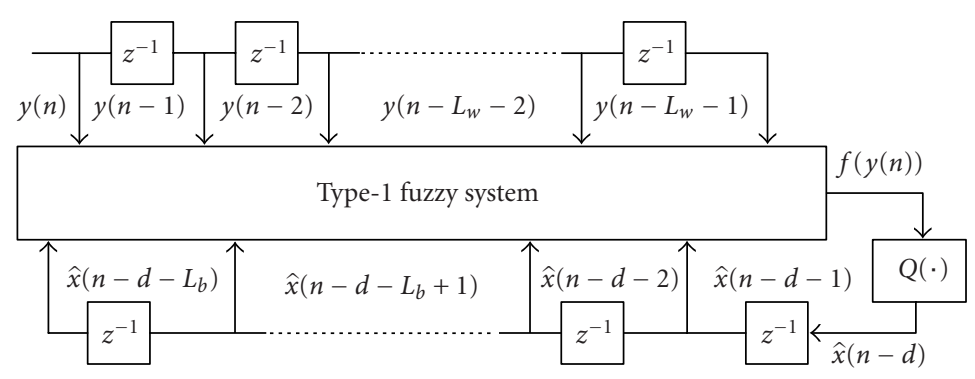

(b)

FIgURE 4: (a) FF structure. (b) DF structure.

It is noticed that the feedback branch reduces the number of channel states required for the decision purposes, as in [29].

It is worth pointing out that the equalization of PL channels is not a simple task to be performed due to the following reasons. (1) PL channel impulse responses for broadband application are long. (2) The use of channel and channel states estimation techniques demands high computational complexity, even though a DF structure is implemented. (3) The loss of optimality of the normalized Bayesian equalizer is frequent if the probability of outlier occurrences is high.

For dealing with these inconveniences, Figure 4 depicts the feedforward (FF) and DF structures of the proposed fuzzy equalizers. For both approaches, the pdf of additive noise in the PL channels is substituted by a nonsingleton fuzzy membership $[14,30]$. The output for both structures is given by

$$
\begin{aligned}
& f(\mathbf{y}(n)) \\
& =\frac{\sum_{l=1}^{M} \theta_{!} \prod_{i=0}^{L-1} \exp \left(-\left(y(n-i)-m_{F_{i}^{l}}\right)^{2} /\left(\sigma_{y}^{2}+\sigma_{F_{i}^{l}}^{2}\right)\right)}{\sum_{l=1}^{M} \prod_{i=0}^{L-1} \exp \left(-\left(y(n-i)-m_{F_{i}^{l}}\right)^{2} /\left(\sigma_{y}^{2}+\sigma_{F_{i}^{l}}^{2}\right)\right)},
\end{aligned}
$$

where $\sigma_{y}^{2}$ is the variance associated to each fuzzy input set, and $\sigma_{F_{i}^{l}}^{2}$ as well as $m_{F_{i}^{l}}$ are the parameters of the Gaussian membership function. The input vectors $\mathbf{y}(n)$ of the FF and DF structures are equal to $\left[\begin{array}{lll}y(n) & \cdots & y\left(n-L_{w}+1\right)\end{array}\right]^{T}$ and $\left[y(n) \cdots y\left(n-L_{w}+1\right) \hat{x}(n-d) \cdots \hat{x}\left(n-d-L_{b}+1\right)\right]^{T}$, respectively. As can be noticed, this model takes into consideration the occurrence of impulse noises. Based upon nonsingleton assumption for PL noise distribution, the normalized and optimal nonsingleton fuzzy equalizer (NONFE) is given by

$$
\begin{aligned}
& f_{\mathrm{bns}}(\mathbf{y}(n)) \\
& =\frac{1}{\sum_{\tilde{\mathbf{y}}_{k} \in \mathbf{C}_{d}} \sum_{i=0}^{L_{w}-1} \exp \left(-\left(y(n-i)-\tilde{y}_{k}(i)\right)^{2} / 2\left(\sigma_{y}^{2}+\sigma_{F_{i}^{k}}^{2}\right)\right)} \\
& \quad \times\left\{\sum_{\tilde{\mathbf{y}}_{k} \in \mathbf{C}_{d}^{++}} \sum_{i=0}^{L_{w}-1} \exp \left(\frac{-\left(y(n-i)-\tilde{y}_{k}(i)\right)^{2}}{2\left(\sigma_{y}^{2}+\sigma_{F_{i}^{k}}^{2}\right)}\right)\right. \\
& \left.\quad-\sum_{\widetilde{\mathbf{y}}_{k} \in \mathbf{C}_{d}^{--}} \sum_{i=0}^{L_{w}-1} \exp \left(\frac{-\left(y(n-i)-\tilde{y}_{k}(i)\right)^{2}}{2\left(\sigma_{y}^{2}+\sigma_{F_{i}^{k}}^{2}\right)}\right)\right\},
\end{aligned}
$$

where $y(n-i)$ and $\tilde{y}_{k}(i)$ are the $i$ th output channel sample and the $i$ th element of the $k$ th output state vector. Note that if $\sigma_{F_{i}^{k}}^{2}$ is equal to a constant $\sigma_{n}^{2}$, then

$$
\left.\lim _{\sigma_{y}^{2} \rightarrow 0} f_{\mathrm{bns}}(\mathbf{y}(n))\right|_{\sigma_{F_{i}^{l}}^{2}=\sigma_{n}^{2}}=f_{b}(\mathbf{y}(n))
$$

The DF version of NONFE is obtained assuming that the equalizer input vector is composed of output channel samples along with past output decisions. In this case, the state matrices $\mathbf{C}_{d}^{--}$and $\mathbf{C}_{d}^{++}$defined by (8) and (9), respectively, substitute $\mathbf{C}_{d}^{-}$and $\mathbf{C}_{d}^{+}$in (12). As a result, the new $\mathbf{C}_{d}$ matrix is equal to $\mathbf{C}_{d}^{--} \cup \mathbf{C}_{d}^{++}$.

These kinds of equalizers also make use of channel or channel state estimation techniques that demand high computational complexity. Although the use of the 
backpropagation method to update the free parameters of these equalizers shows low computational complexity, it has low convergence rate and often yields suboptimal solutions. In this case, the use of updating step size techniques along with the backpropagation method may be an interesting solution to improve the convergence rate.

In this regard, DBD [15] and LLE [16] methods can be good candidates for updating the step size associated with each individual free parameter. These methods provide high convergence rates as they try to find the proper learning rate to compensate small magnitude of the gradient in the flat regions and to dampen the large free parameter changes in high-depth regions. From the author's point of view, these methods can be considered as a modified version of the backpropagation method.

Regarding the first method, it is known that the DBD learning rule consists of a parameter vector updating rule performed by a modified backpropagation procedure and a learning rate rule defined by

$$
\begin{aligned}
\Delta \mathbf{w}(n+1)= & -(1-\alpha) \operatorname{diag}\left\{\mu_{0}(n), \ldots, \mu_{P-1}(n)\right\} \\
& \times \nabla J(\mathbf{w}(n))+\alpha \Delta \mathbf{w}(n), \\
\mu_{i}(n+1)= & \begin{cases}\kappa & \text { if } \bar{\lambda}_{i}(n-1) \lambda_{i}(n)>0, \\
-\phi \mu_{i}(n) & \text { if } \bar{\lambda}_{i}(n-1) \lambda_{i}(n)<0, \\
0 & \text { otherwise, }\end{cases}
\end{aligned}
$$

respectively, where $i=0, \ldots, P-1$,

$$
\mathbf{w}(n)=\left[\begin{array}{lll}
w_{0}(n) & \cdots & w_{P-1}(n)
\end{array}\right]^{T}
$$

denotes the free parameter vector of a specific fuzzy equalizer, $\boldsymbol{\mu}(n)=\left[\begin{array}{lll}\mu_{0}(n) & \cdots & \mu_{P-1}(n)\end{array}\right]^{T}$ is the learning rate vector, $\Delta \mathbf{w}(n+1)=\mathbf{w}(n+1)-\mathbf{w}(n), \alpha$ is the momentum rate, $\lambda_{i}(n)=\partial J(\mathbf{w}(n)) / \partial w_{i}(n)$ is the partial derivative of the cost function with respect to $w_{i}(n)$ at the $n$th iteration, and $\bar{\lambda}(n)=(1-\delta) \lambda(n)+\delta \bar{\lambda}(n-1)$ is an exponential average of the current and past derivatives.

Considering the second method, it is established that the LLE method, in turn, is based on the estimation of the local Lipschitz constant $\Lambda$ in each free parameter direction [16]. As far as adaptive fuzzy systems are concerned, neither the morphology of the error surface nor the values of $\Lambda$ are known a priori. Then the estimation of $\Lambda$ is obtained from the maximum (infinity) norm given by

$$
\widehat{\Lambda}(n+1)=\frac{\max _{0 \leq i \leq P-1}\left|\nabla J_{i}(\mathbf{w}(n+1))-\nabla J_{i}(\mathbf{w}(n))\right|}{\max _{0 \leq i \leq P-1}\left|\mathbf{w}_{i}(n+1)-\mathbf{w}_{i}(n)\right|} .
$$

As the shape of error surface to adapt a specific step size $\mu_{i}=$ $1 / \Lambda_{i}(n+1), 0 \leq i \leq P-1$, for each weight estimated in the $i$ th parameter direction, the fuzzy free parameters updating
TABLE 1: Additional computational cost associated with DBD and LLE methods.

\begin{tabular}{lll}
\hline $\begin{array}{l}\text { Computational } \\
\text { complexity }\end{array}$ & $\mathrm{DBD}$ & $\mathrm{LLE}$ \\
\hline Addition & $\mathrm{Ca}(\mathrm{BP})+\mathrm{P}$ & $\mathrm{Ca}(\mathrm{BP})+\mathrm{P}$ \\
Subtraction & $\mathrm{Cs}(\mathrm{BP})+2 \mathrm{P}$ & $\mathrm{Cs}(\mathrm{BP})+3 \mathrm{P}$ \\
Multiplication & $\mathrm{Cm}(\mathrm{BP})+3 \mathrm{P}$ & $\mathrm{Cm}(\mathrm{BP})+3 \mathrm{P}$ \\
Division & $\mathrm{Cd}(\mathrm{BP})$ & $\mathrm{Cd}(\mathrm{BP})+2 \mathrm{P}$ \\
Comparison & $3 \mathrm{P}$ & 1 \\
\hline
\end{tabular}

rule is given by

$$
\begin{aligned}
& \Delta \mathbf{w}(n+1) \\
& \quad=-\lambda(n) \operatorname{diag}\left\{\mu_{0}(n+1), \ldots, \quad \mu_{P-1}(n+1)\right\} \nabla J(\mathbf{w}(n)), \\
& \mu_{i}(n+1)=\frac{1}{\Lambda_{i}(n+1)} \\
& =\frac{\left|\mathbf{w}_{i}(n+1)-\mathbf{w}_{i}(n)\right|}{\left|\nabla J_{i}(\mathbf{w}(n+1))-\nabla J_{i}(\mathbf{w}(n))\right|}, \quad i=0, \ldots, P-1,
\end{aligned}
$$

where the relaxation coefficient $\lambda(n)$ must satisfy the following condition:

$$
\begin{aligned}
& \nabla J_{i}(\mathbf{w}(n+1))-\nabla J_{i}(\mathbf{w}(n)) \\
& \leq-\frac{1}{2} \lambda(n)\left\|\operatorname{diag}\left\{\mu_{0}(n+1), \ldots, \mu_{P-1}(n+1)\right\} \nabla J(\mathbf{w}(n))\right\|^{2} .
\end{aligned}
$$

The following rule is evaluated to update $\lambda(n)$.

If (18) is true, then

$$
m=m-1, \quad \lambda(n+1)=\frac{\lambda_{0}}{q^{m-1}}
$$

otherwise

$$
m=m+1, \quad \lambda(n+1)=\frac{\lambda_{0}}{q^{m-1}}
$$

where $q \in \mathfrak{R}$ denotes the reduction factor, $\lambda_{0}$ is the initial relaxation coefficient, and $m$ is a positive integer number. The computational cost per iteration associated with the DBD and LLE methods is shown in Table 1. The total number of free parameters $P$ is expressed by

$$
P= \begin{cases}M(2 L+1)+1 & \text { if nonsingleton, } \\ M(2 L+1) & \text { if singleton }\end{cases}
$$

where

$$
L= \begin{cases}L_{w} & \text { if FF structure } \\ L_{w}+L_{b} & \text { if DF structure. }\end{cases}
$$


In Table 1, Ca (BP), Cs (BP), Cm (BP), and $C d$ (BP) represent the computational complexity of the backpropagation method in terms of the number of additions, subtractions, multiplications, and divisions, respectively. Note, in Table 1, that the computational complexity increments due to DRD and LLE methods have been evaluated based on computational complexity of the traditional backpropagation method. From Table 1, it can be stated that by using a hardware solution (DSP or FPGA), a linear increase in the computational complexity per iteration is observed when the DRD and LLE methods are applied for training fuzzy equalizers. Section 4 shows some results illustrating that this linear increase of computational complexity can significantly improve the convergence rate. As a result, the fuzzy equalizers can be applied for periodical PL channel equalizations.

\section{SIMULATION RESULTS}

In this section, the convergence rate of the proposed fuzzy equalizers called fuzzy-S-LMS-DRD, fuzzy-S-LMS-LLE, fuzzy-S-DFE-DRD, fuzzy-S-DFE-LLE, fuzzy-NS-LMS-DRD, fuzzy-NS-LMS-LLE, fuzzy-NS-DFE-DRD, and fuzzy-NSDFE-LLE are compared, under severe noise scenario, to the previous equalizers which we name fuzzy-S-LMS, fuzzy-SDFE, fuzzy-NS-LMS, and fuzzy-NS-DFE [12, 13, 14, 24, 30]. For simplicity, only the results attained by using fuzzy-SDFE-LLE and fuzzy-NS-DFE-LLE equalizers are illustrated in terms of BER performance.

The chosen PL channel and impulse noise models are drawn from $[6,7]$, respectively. To obtain the BER curve, the following considerations are observed: (a) the PL channel is normalized; (b) the frequency range is between $1 \mathrm{MHz}$ and $2.5 \mathrm{MHz}$; (c) the power of the transmitted BPSK symbols and the impulse noise are equal to $\sigma_{x}^{2}=0 \mathrm{~dB}$ and $\sigma_{v_{\text {imp }}}^{2}=0 \mathrm{~dB}$, respectively; (d) the power of background noise varies from $-2.5 \mathrm{~dB}$ to $-20 \mathrm{~dB}$; (e) $L_{w}, L_{b}, M$, and $d$ are equal to 15 , 8,100 , and 0 , respectively; (f) the step size for the previous fuzzy equalizer is equal to $0.001 ;(\mathrm{g}) \alpha \in[0.1,0.4]$, $\kappa \in[0.001,0.0001], \phi \in[0.6,1.0]$, and the initial step size is equal 0.03 ; (h) $\lambda_{0}, m$, and $q$ are equal to 4,1 , and 1.038 , respectively; (i) the same free parameter initialization conditions were applied to all analyzed equalizers.

The convergence rates of the proposed FF and DF equalizers in terms of MSE measure when $\sigma_{x}^{2}=0 \mathrm{~dB}, \sigma_{v_{i n}}^{2}=0 \mathrm{~dB}$, and $\sigma_{\mathrm{bkgr}}^{2}=-20 \mathrm{~dB}$ are shown in Figures 5 and 6 , respectively. As noted, the new techniques attain lower MSE values with a smaller number of iterations than the previous fuzzy equalizers. It is worth stating that all fuzzy equalizers with the same structure will converge to the same MSE. The faster convergence rate of the NS-LLE proposals is due to two reasons.

The first reason refers to the fact that the nonsingleton versions show at least the same convergence rate as their equivalent singleton equalizers. In fact, the nonsingleton fuzzy equalizers deal with the uncertainty in the input and, as a result, are able to mitigate the presence of impulse noises more easily.

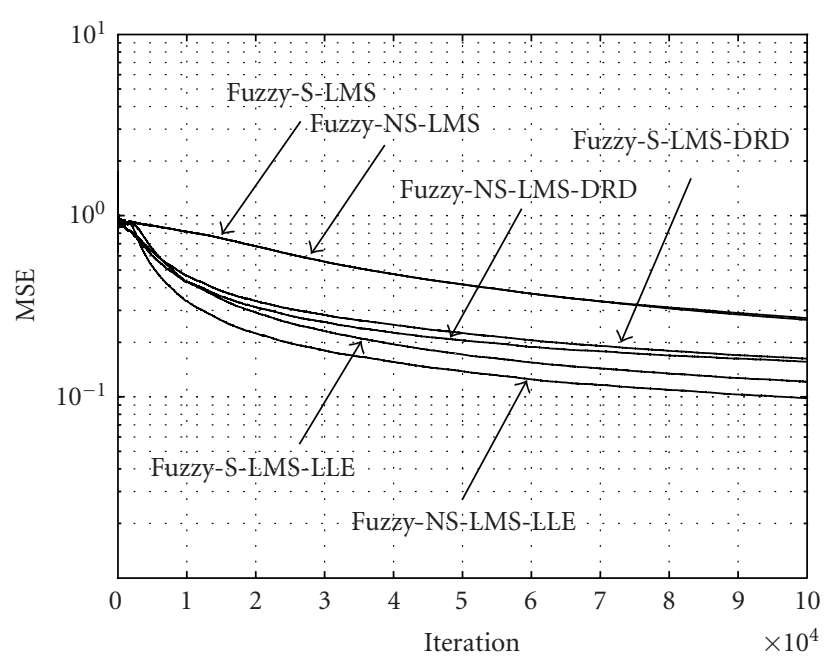

FIGURE 5: FF fuzzy equalizers.

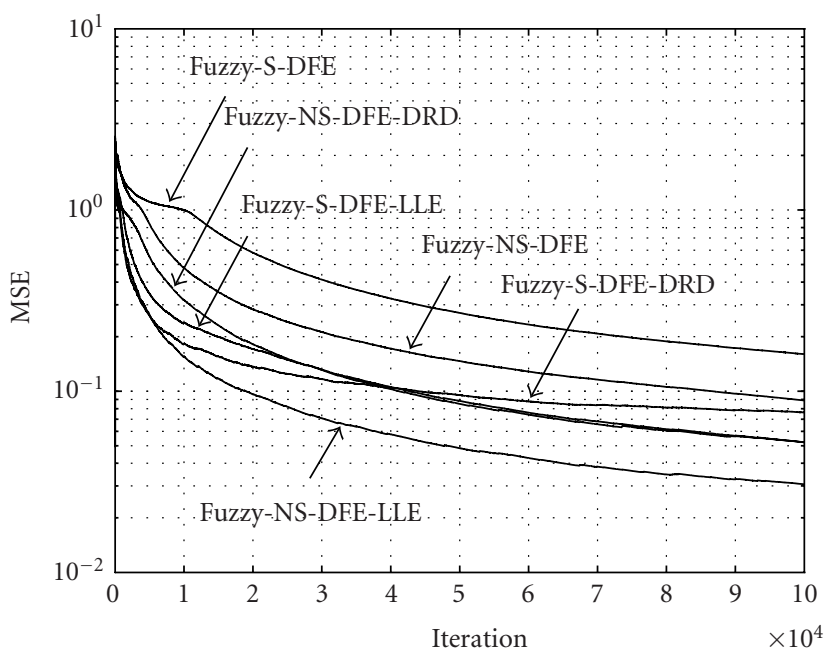

Figure 6: DF fuzzy equalizers.

The second reason refers to the efficiency of the training method applied to fuzzy equalizers, which deserves considerable attention.

Figures 5 and 6 show that the LLE and DRD methods provide the highest convergence rate while the use of the traditional backpropagation methods shows the lowest convergence rate. Although more computational complexity per iteration is demanded by LLE and DRD methods (see Table 1) the gain in terms of convergence rate is 5 times as high when compared to fuzzy equalizers trained by backpropagation method.

Figures 7 and 8 portray the BER performance of the fuzzy-S-DFE-LLE, fuzzy-NS-DFE-LLE, DFE [28], and Bayesian (optimal) equalizers [19] with and without error propagation, respectively. The SNR values in these graphs represent the relation between the power of the transmitted symbols and the power of the background noises. Also, the impulse noise power $\sigma_{v_{\text {in }}}^{2}=0 \mathrm{~dB}$ was considered to configure 


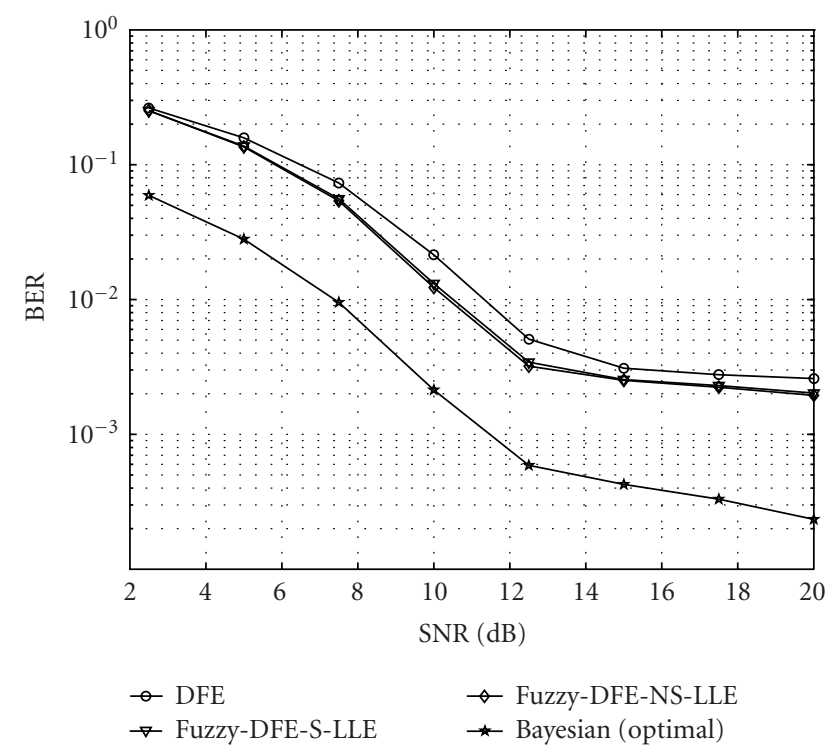

FIGURE 7: BER performance of DF equalizers with error propagation.

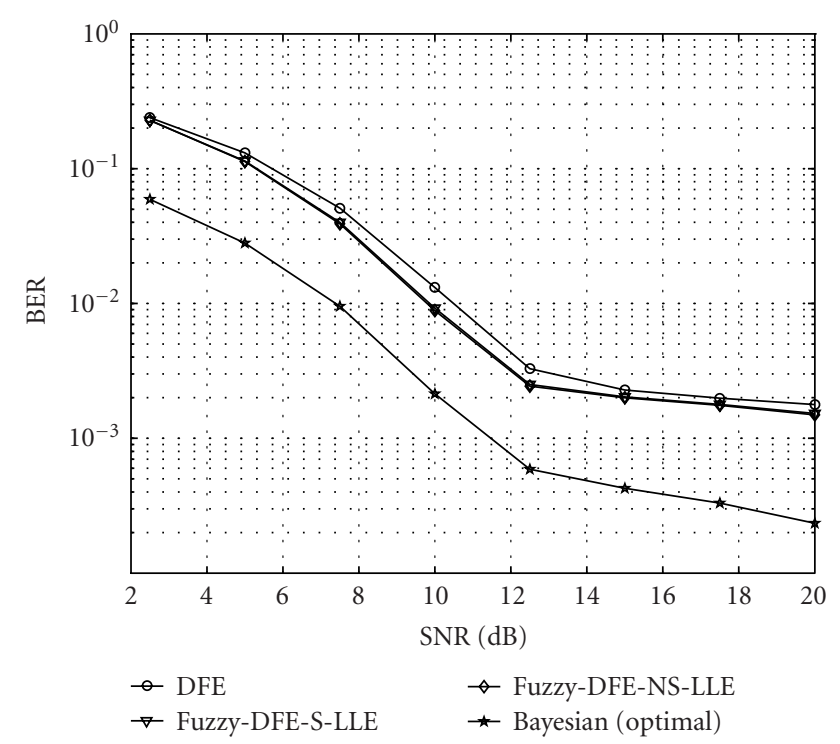

FIGURE 8: BER performance of DF equalizers without error propagation.

a harsh PLC scenario. To get these numerical results, the number of iterations ranged from $2 \times 10^{6}$ to $10^{7}$.

As can be observed, the proposed equalizers exhibit a better performance than traditional DF equalizers. Traditional fuzzy equalizers can also attain these results. However, this demands at least 4 times the number of iterations spent to obtain the convergence of the fuzzy-S-DFE-LLE and fuzzyNS-DFE-LLE equalizers. Although the BER performance of the FF versions was not shown in this work, it is worth mentioning that it shows the worst results due to their innate features.

\section{CONCLUSIONS}

This contribution has addressed the use of learning rate updating methods to increase the convergence rate of the adaptive fuzzy equalizers. On the basis of the results attained, we can conclude that the proposed equalizers are a satisfactory alternative solution to mitigate the hardness of ISI and impulse noise effects for broadband PLC applications. The computational results appropriately illustrate the applicability of these adaptive fuzzy equalizers revealing that they are a new means of achieving high-rate transmissions at lower BER in PLC systems. Furthermore, they demand fewer iterations than traditional fuzzy equalizers to converge.

Further investigations are being carried out to analyze the use of type-2 fuzzy systems with updating step size and to extend the analysis to other constellations. Another interesting investigation is the use of the proposed fuzzy equalizers in a turbo equalization scheme (see [31]) to reduce the number of turbo iterations required by the turbo fuzzy equalizer convergence.

\section{ACKNOWLEDGMENTS}

We are sincerely indebted to the anonymous reviewers for their valuable suggestions and comments. Special thanks are extended to Patrícia N. S. Ribeiro for proofreading this contribution. The authors are also thankful to CAPES (BEX2418/03-7), CNPq (Grant 552371/01-7), and FAPESP (Grants 01/08513-0 and 02/12216-3) from Brazil for their financial support.

\section{REFERENCES}

[1] N. Pavlidou, A. J. Han Vinck, J. Yazdani, and B. Honary, "Power line communications: state of the art and future trends," IEEE Communications Magazine, vol. 41, no. 4, pp. 34-40, 2003.

[2] J. Abad, A. Badenes, J. Blasco, et al., "Extending the power line LAN up to the neighborhood transformer," IEEE Communications Magazine, vol. 41, no. 4, pp. 64-70, 2003.

[3] A. J. Han Vinck and G. Lindell, "Summary of contributions at the international symposium on power line communications and its applications," in Proc. 5th International Symposium on Power Line Communications and Its Applications (ISPLC '01), pp. 383-413, Malmö, Sweden, April 2001.

[4] F. J. Cañete, J. A. Cortes, L. Diez, and J. T. Entrambasaguas, "Modeling and evaluation of the indoor power line transmission medium," IEEE Communications Magazine, vol. 41, no. 4, pp. 41-47, 2003.

[5] F. J. Cañete, L. Diez, J. A. Cortes, and J. T. Entrambasaguas, "Broadband modelling of indoor power-line channels," IEEE Transactions on Consumer Electronics, vol. 48, no. 1, pp. 175$183,2002$.

[6] M. Zimmermann and K. Dostert, "A multipath model for the powerline channel," IEEE Trans. Communications, vol. 50, no. 4, pp. 553-559, 2002.

[7] M. Zimmermann and K. Dostert, "Analysis and modeling of impulsive noise in broad-band powerline communications," IEEE Transactions on Electromagnetic Compatibility, vol. 44, no. 1, pp. 249-258, 2002. 
[8] P. J. Langfeld, M. Zimmermann, and K. Dostert, "Power line communication system design strategies for local loop access," in Proc. Workshop Kommunikationstechnik, pp. 21-26, Ulm, Germany, July 1999.

[9] P. J. Langfeld, "The capacity of typical power line reference channels and strategies for system design," in Proc. 5th International Symposium on Power Line Communications and Its Applications (ISPLC '01), pp. 271-278, Malmö, Sweden, April 2001.

[10] Ascom Power Line Communications AEG, "Nothing is as powerful as an idea for which the time is ripe: Welcome to the second discovery of electricity!," June 2001, www.ascom.com.

[11] S. Qureshi, "Adaptive equalization," Proceedings of the IEEE, vol. 73, no. 9, pp. 1349-1387, 1985.

[12] M. V. Ribeiro, "On fuzzy-DFE-LMS and fuzzy-DFE-RLS algorithms to equalize power line channels," in Proc. IEEE International Symposium on Industrial Electronics (ISIE '03), vol. 2, pp. 1001-1006, Rio de Janeiro, Brazil, June 2003.

[13] L.-X. Wang and J. M. Mendel, "Fuzzy adaptive filters, with application to nonlinear channel equalization," IEEE Transactions on Fuzzy Systems, vol. 1, no. 3, pp. 161-170, 1993.

[14] J. M. Mendel, Uncertain Rule-Based Fuzzy Logic Systems: Introduction and New Directions, Prentice-Hall, Upper Saddle River, NJ, USA, 2001.

[15] R. A. Jacobs, "Increased rates of convergence through learning rate adaptation," Neural Networks, vol. 1, no. 4, pp. 295-307, 1988.

[16] G. D. Magoulas, M. N. Vrahatis, and G. S. Androulakis, "Improving the convergence of the backpropagation algorithm using learning rate adaptation methods," Neural Computation, vol. 11, no. 7, pp. 1769-1796, 1999.

[17] G. D. Forney Jr., "Maximum-likelihood sequence estimation of digital sequences in the presence of intersymbol interference," IEEE Transactions on Information Theory, vol. 18, no. 3, pp. 363-378, 1972.

[18] G. D. Forney Jr., "The Viterbi algorithm," Proceedings of the IEEE, vol. 61, no. 3, pp. 268-278, 1973.

[19] R. O. Duda and P. E. Hart, Pattern Classification and Scene Analysis, John Wiley \& Sons, New York, NY, USA, 1973.

[20] D. E. Rumelhart, G. E. Hinton, and R. J. Williams, "Learning internal representations by error propagation," in Parallel Distributed Processing: Explorations in the Microstructure of Cognition, vol. 1, pp. 318-362, MIT Press, Cambridge, Mass, USA, 1986.

[21] T. C. Chuah, B. S. Sharif, and O. R. Hinton, "Robust adaptive spread-spectrum receiver with neural net preprocessing in non-Gaussian noise," IEEE Transactions on Neural Networks, vol. 12, no. 3, pp. 546-558, 2001.

[22] S. Chen, G. J. Gibson, C. F. N. Cowan, and P. M. Grant, "Adaptive equalization of finite non-linear channels using multilayer perceptrons," Signal Processing, vol. 20, no. 2, pp. 107119, 1990.

[23] Q. Liang and J. M. Mendel, "Equalization of nonlinear timevarying channels using type-2 fuzzy adaptive filters," IEEE Transactions on Fuzzy Systems, vol. 8, no. 5, pp. 551-563, 2000.

[24] S. K. Patra and B. Mulgrew, "Fuzzy techniques for adaptive nonlinear equalization," Signal Processing, vol. 80, no. 6, pp. 985-1000, 2000.

[25] S. K. Patra and B. Mulgrew, "Efficient architecture for Bayesian equalization using fuzzy filters," IEEE Trans. on Circuits and Systems II: Analog and Digital Signal Processing, vol. 45, no. 7, pp. 812-820, 1998.

[26] M.-S. Yee, B. L. Yeap, and L. Hanzo, "Radial basis functionassisted turbo equalization," IEEE Trans. Communications, vol. 51, no. 4, pp. 664-675, 2003.
[27] E.-S. Chng, H. Yang, and W. Skarbek, "Reduced complexity implementation of Bayesian equaliser using local RBF network for channel equalisation problem," Electronics Letters, vol. 32, no. 1, pp. 17-19, 1996.

[28] M. E. Austin, "Decision-feedback equalization for digital communication over dispersive channels," Tech. Rep. 437, Research Laboratory of Electronics, MIT, Cambridge, Mass, USA, August 1967.

[29] S. Chen, B. Mulgrew, and S. McLaughlin, "Adaptive Bayesian equalizer with decision feedback," IEEE Trans. Signal Processing, vol. 41, no. 9, pp. 2918-2927, 1993.

[30] G. C. Mouzouris and J. M. Mendel, "Nonsingleton fuzzy logic systems: theory and application," IEEE Transactions on Fuzzy Systems, vol. 5, no. 1, pp. 56-71, 1997.

[31] M. B. Loiola, M. V. Ribeiro, and J. M. T. Romano, "A turbo equalizer using fuzzy filters," in Proc. IEEE International Workshop on Machine Learning for Signal Processing (MLSP '04), São Luís, Brazil, September/October 2004.

Moisés V. Ribeiro was born in Três Rios, Brazil, in 1974. He received the B.S. degree from the Federal University of Juiz de Fora, Brazil, in 1999, and the M.S. degree from the State University of Campinas (UNICAMP), Campinas, Brazil, in 2001, both in electrical engineering. He is currently working toward the Ph.D. degree at UNICAMP. Mr. Ribeiro was a Visiting Researcher in the Image and Signal Processing Laboratory of the University of California, Santa Barbara, from January 2004 to June 2004. He holds one patent. His fields of interests include filter banks, computational intelligence, digital and adaptive signal processing applied to power quality evaluation, and power line communication. He was granted Student Awards by IECON '01 and ISIE '03. 